\title{
Direct Numerical Simulations of Grouping Effects in Droplet Streams Using Different Boundary Conditions
}

\author{
Matthias Ibach*1, Kathrin Schulte ${ }^{1}$, Visakh Vaikuntanathan ${ }^{1}$, Alumah Arad $^{2}$, \\ David Katoshevski ${ }^{2}$, J. Barry Greenberg ${ }^{3}$, Bernhard Weigand ${ }^{1}$ \\ ${ }^{1}$ Institute of Aerospace Thermodynamics (ITLR), University of Stuttgart, Stuttgart, Germany \\ ${ }^{2}$ Environmental Engineering Unit, Ben-Gurion University of the Negev (BGU), \\ Beer-Sheva, Israel \\ ${ }^{3}$ Aerospace Engineering, Technion - Israel Institute of Technology, Haifa, Israel \\ ${ }^{*}$ Corresponding author email: matthias.ibach@itlr.uni-stuttgart.de
}

\begin{abstract}
The tendency of convening and coalescing of initially distant droplets, called droplet grouping, can influence the final droplet size distribution, evaporation rates or final settling points. To better understand the factors which govern droplet grouping mechanisms, reliable direct numerical simulations are beneficial. Previous numerical studies have shown that the approaching and collision process of droplets in a stream is extremely sensitive to the applied boundary conditions. Therefore, simulations are performed regarding the influence of domain size and boundary conditions on the grouping behavior. Improved boundary conditions, so called artificial boundary conditions, are derived to allow for suitable velocity approximations in the far field. A comparison to the existing boundary conditions regarding velocity profiles and grouping behavior for different domain widths and Reynolds numbers is investigated. The proposed artificial boundary condition takes into account the decay of velocities near the side boundaries more accurately than the already implemented boundary conditions in combination with periodicity in the direction of droplet motion. Evaluating droplet spacing over time reveals that the domain width using artificial boundaries can be reduced without influencing the grouping behavior.
\end{abstract}

\section{Keywords}

droplet dynamics, droplet grouping, DNS, artificial boundary condition

\section{Introduction}

The dynamics of liquid droplets plays an important role in dense sprays or droplet streams occurring in various technical systems comprising injection/combustion, coating, medical and agricultural applications [1]. To improve system efficiency the exact droplet sizes and the related evaporation rates, their corresponding size distribution, droplet velocities, the applied drag force and final settling points need to be exactly known. These characteristics, however, can be affected by the tendency of initially distant and isolated droplets to approach each other, coalesce and form larger droplets and groups.

This tendency, called droplet grouping, can arise for example when groups of droplets are traveling in an unsteady host gas environment. Katoshevski et al. [2] reported on clustering (or grouping) in aerosols/sprays in an oscillating flow field with a tendency to form groups depending, among others, on oscillation frequency and droplet size. Recent experiments revealed that grouping can also occur even if droplets are injected into a quiescent ambient environment, for example by a droplet stream generator [3]. In the subsequent work of Roth et al. [4] grouping of droplets was studied in monodisperse droplet streams. By using two frequency generators and manipulating the excitation frequency groups consisting of two droplets with adjustable inter droplet spacing were generated. Roth and colleagues observed that the velocity of the droplet approach depends linearly on the spacing between the droplets. Subsequently performed direct numerical simulations (DNS), with a computational setup based on the conditions of the 
conducted experiments, showed the same linear trend of droplet behavior. However, the time until droplets coalesce could not be reproduced accurately. Problems were attributed to the numerical stability of the simulation and the prescribed lateral boundary conditions (BCs). The droplet stream could be stabilized using walls with no-slip conditions as side boundaries which is of course in contrast to the experiments. Increasing the computational domain resulted in a better approximation, yet, increased computational costs and could still not entirely fit the time of droplet coagulation of the experimental results.

In the following, we present more suitable lateral boundary conditions for a stable numerical setup for the investigation of grouping effects in a droplet stream. Similar to [5] suitable approximations for the far field are used, called artificial boundary conditions (ABCs), which take into account the decay of velocities near the side boundaries more accurately. The simulations are conducted with the multiphase DNS code Free Surface 3D (FS3D) which is described in more detail in the following section along with the computational setup. Following, the implementation into the code framework of the ABCs adapted for droplet streams based on an analytical solution is explained. Several simulations with different boundary conditions and lateral domain widths are conducted. As basis for the validation of the influence on the grouping behavior the experimental data provided by Roth et al. [4] is used. Subsequently performed simulations at different Reynolds numbers investigate the grouping tendency applying the newly implemented ABCs. Finally, the work is summarized and concluded.

\section{Fundamentals}

Numerical method

For the setup and the simulation of grouping effects in droplet streams the ITLR in-house DNS code FS3D for multiphase flows with incompressible fluids is used. The underlying conservation equations are discretized by finite volumes in space on a Cartesian staggered grid and read

$$
\partial_{t} \rho+\nabla \cdot(\rho \mathbf{u})=0
$$

for the mass conservation and

$$
\partial_{t}(\rho \mathbf{u})+\nabla \cdot[(\rho \mathbf{u}) \otimes \mathbf{u}]=\nabla \cdot[\mathbf{S}-\mathbf{I} p]+\rho \mathbf{g}+\mathbf{f}_{\gamma}
$$

for the momentum conservation with $\rho$ denoting the density, $t$ the time, $\mathbf{u}$ the velocity vector, $\mathbf{S}$ the viscous stress tensor (defined as $\mathbf{S}=\mu\left[\nabla \mathbf{u}+\nabla \mathbf{u}^{T}\right]$ with $\mu$ as constant dynamic viscosity), I the identity matrix and $p$ the pressure. Other body forces such as gravity or surface tension are represented by $\mathrm{g}$ and $\mathbf{f}_{\gamma}$, respectively.

The flow field is computed by solving equations (1) and (2) in a one-field formulation where the different phases are regarded as a single fluid with variable physical properties. In order to identify the different phases, namely the continuous (here the surrrounding gaseous phase, subscript $g$ ) and the disperse or liquid phase (subscript $l$ ), an additional indicator variable $f(\mathbf{x}, t)$ is introduced according to the classical Volume of Fluid method (VOF) developed by Hirt and Nichols [6]. This scalar is defined as being 0 in the continuous and 1 in the disperse phase and taking values between $(0,1)$ indicating the presence of an interface. The advection of the scalar $f$ across the computational domain is achieved by the transport equation

$$
\partial_{t} f+\nabla \cdot(f \mathbf{u})=0 .
$$

Consistent with the one-field formulation local material properties such as the density (or the viscosity) are calculated following the expression

$$
\rho(\mathbf{x}, t)=\rho_{g}(1-f(\mathbf{x}, t))+\rho_{l} f(\mathbf{x}, t) .
$$

To reduce numerical diffusion for the advection of the scalar $f$ FS3D employs the Piecewise Linear Interface Calculation (PLIC) algorithm according to Rider and Kothe [7] to correct the numerical fluxes in interface cells. The continuous surface stress (CSS) model by 
Lafaurie et al. [8] is used for the consideration of the surface tension force $\mathbf{f}_{\gamma}$ in the performed simulations.

Especially in terms of fluid dynamics the code is well validated. More recent studies extended the applicability to free falling droplets in turbulent surroundings [9], droplet impact onto a thin film [10] or structured surfaces [11], robust methods for phase change processes [12] as well as droplet interaction with multiple species [13] with the latter two aspects being also interesting to grouping effects in droplet streams. In order to cope with the demanding requirements of DNS FS3D is fully parallelized using MPI and OpenMP. The code has been adapted to run on supercomputers such as the HPE Apollo (Hawk) system at the High Performance Computing Center Stuttgart (HLRS) where the presented simulations were conducted. For a more detailed overview of FS3D and its capabilities the reader is referred to [14].

\section{Computational setup}

For the simulation of the droplet stream four droplets are placed in the center of the computational domain with two slightly different spacings $a_{i}$ between the droplets in order to take into account the two pairs created in the experiment. The different inter droplet spacings indicate the distance between the last droplet of a pair and the first droplet of the following pair $a_{1}$ and the spacing between the droplets of a single pair $a_{2}$ (see figure 1 (a)). The distance between a group is defined as $g=a_{1}+a_{2}$. All four droplets are initialized with the same initial velocity $u_{0}$

(a)

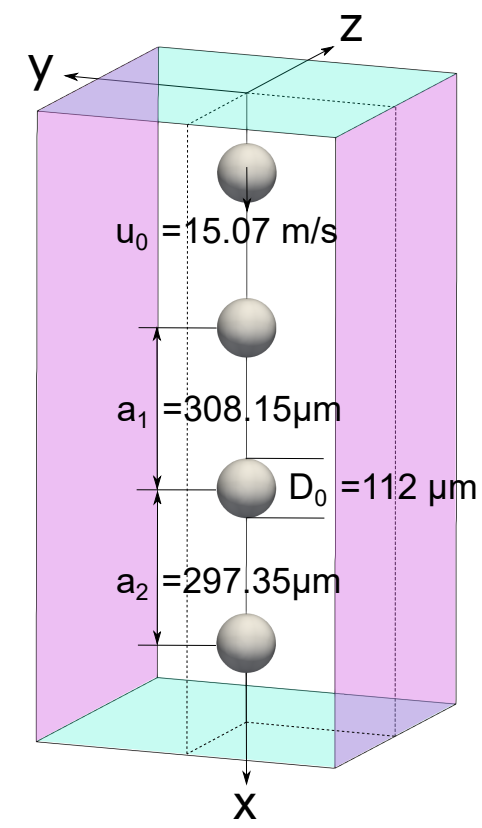

(b)

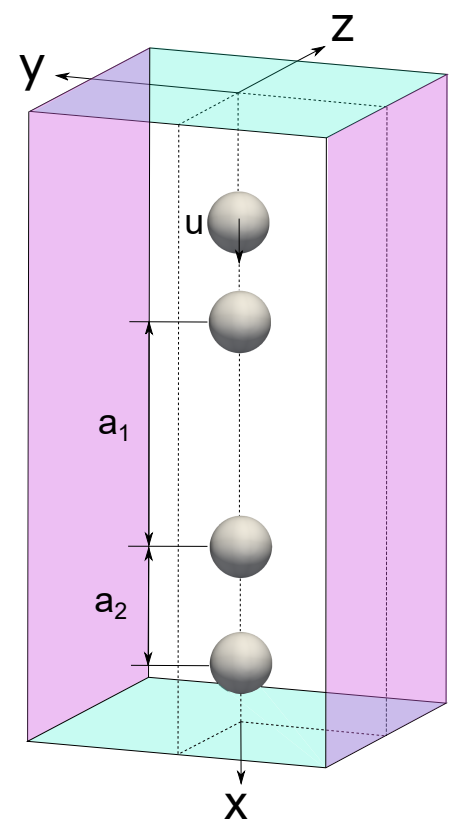

Figure 1. Initial setup of a droplet stream with exemplary domain width of $y / D_{0}=z / D_{0}=2.7$, periodic (light blue) and other settable boundary conditions (light magenta) (a) and visible grouping effect at $t=4 \mathrm{~ms}$ (b).

with the gravitational force pointing in the direction of droplet motion. The initial conditions of the iso-propanol droplets at ambient pressure and temperature (droplet diameter $D_{0}$, inter droplet distance $a_{i}$ and initial velocity $u_{0}$ ) are set in correspondance to the experimental work of Roth et al. [4] and are summarized in table 1 along with the nondimensional parameters $R e=\left(\rho_{g} u_{0} D_{0}\right) / \mu_{g}, W e=\left(\rho_{g} u_{0}^{2} D_{0}\right) / \sigma$ and $O h=\mu_{l} / \sqrt{\rho_{l} \sigma D_{0}}$.

Table 1. Operating conditions and fluid properties of iso-propanol acoording to [4].

\begin{tabular}{cccccccc}
\hline \hline $\begin{array}{c}R e \\
{[-]}\end{array}$ & $\begin{array}{c}W e \\
{[-]}\end{array}$ & $\begin{array}{c}O h \\
{[-]}\end{array}$ & $\begin{array}{c}\rho_{l} \\
{\left[\mathrm{~kg} \mathrm{~m}^{-3}\right]}\end{array}$ & $\begin{array}{c}\rho_{g} \\
{\left[\mathrm{~kg} \mathrm{~m}^{-3}\right]}\end{array}$ & $\begin{array}{c}\mu_{l} \\
{[\mathrm{mPa} \mathrm{s}]}\end{array}$ & $\begin{array}{c}\mu_{g} \\
{[\mathrm{mPa} \mathrm{s}]}\end{array}$ & $\begin{array}{c}\sigma \\
{\left[\mathrm{mN} \mathrm{m}^{-1}\right]}\end{array}$ \\
\hline 110.6 & 1.438 & 0.05242 & 786 & 1.204 & 2.270 & 0.01837 & 21.30 \\
\hline \hline
\end{tabular}


The computational domain consists of a regular Cartesian grid where the lateral distances of the boundary domains $y / D_{0}$ and $z / D_{0}$ are varied according to the investigated case. The dashed lines indicate the possibility to simulate only one quarter of the domain and take into account the symmetry of the problem at moderate Reynolds numbers. The droplets are resolved with 24 grid cells per diameter which is in accordance to previous numerical work. To mirror the behavior of a continuous droplet stream such as in the experiment, periodic boundaries in the direction of motion are applied (light blue boundaries in figure 1). All remaining boundaries can be set to slip (also used for symmetry planes), no-slip (impermeable wall) and zero Neumann BCs (continuous type) indicated exemplarily by the light magenta boundary in the $x$-z-plane. The results with the currently implemented and the newly proposed artificial boundary conditions in FS3D are explained in more detail in the following section.

\section{Results and discussion}

\section{Boundary conditions}

Flows around fluid particles often need to be regarded as a problem within infinite domains to avoid the influence of domain walls. Numerical simulations, however, demand the modeling with a bounded domain. Moreover, a high resolution in DNS usually requires a large number of computational cells. To reduce the computational effort the domain has to be as small as possible. No-slip or slip boundary conditions are often the choice of preference (equation (5)), which, however, do not account for the decay behavior of the velocity field with increasing lateral distance from the droplet stream.

$$
\begin{array}{ll}
u_{n}=0 & \text { and } u_{\tau}=0 \quad \text { for no-slip boundary conditions / walls } \\
u_{n}=\left\{0, u_{n}\right\} & \text { and } \partial_{n} u_{\tau}=0 \text { for slip or continuous boundary conditions }
\end{array}
$$

Previous studies such as droplet oscillations or droplet impacts with FS3D have shown that the available boundary conditions are generally suitable for larger domain widths and the related amount of computational calculation cycles. For the simulation of infinite droplet streams, implying periodic boundaries, the currently implemented BCs do not lead to satisfying results: An asymmetric influence on the flow field due to the influence of the boundary domains results in a drift of the droplet chain towards the side boundaries. The droplets can be stabilized by making use of the symmetry of the problem or applying no-slip conditions at the lateral sides (see also [4]). This is, however, in contrast to the experiment and changes the behavior of the droplets drastically, thus the idea to introduce artificial boundary conditions.

In FS3D boundary conditions are realized with dummy cells implying two additional layers of cells outside the physical domain. Adding these cells the same spatial discretization scheme can be employed at the boundary as inside the domain. The boundary conditions are instantiated by setting appropriate dummy values and derivatives in normal or tangential direction in those cells. The outer velocity field of the analytical Hadamard-Rybczynski $(H-R)$ solution serves as the basis for the current approach for the ABCs [15]. Equation (6) describes the velocity components $u_{r}(r, \theta)$ in radial and $u_{\theta}(r, \theta)$ in tangential direction employing spherical coordinates (axially symmetric around $z$-axis, azimuthal angle $\varphi$ can be omitted) with suitable coefficients $\alpha_{1-3}$ :

$$
\begin{aligned}
& u_{r}(r, \theta)=\left(\frac{\alpha_{1}}{r^{3}}+\frac{\alpha_{2}}{r}+\alpha_{3}\right) \cos (\theta) \quad u_{\theta}(r, \theta)=\left(\frac{1}{2} \frac{\alpha_{1}}{r^{3}}-\frac{1}{2} \frac{\alpha_{2}}{r}-\alpha_{3}\right) \sin (\theta) \\
& \alpha_{1}=R_{0}^{3}\left(-\frac{u_{0}}{2} \frac{\mu_{l} / \mu_{g}}{1+\mu_{l} / \mu_{g}}\right) \quad \alpha_{2}=R_{0}\left(\frac{u_{0}}{2} \frac{2+3 \mu_{l} / \mu_{g}}{1+\mu_{l} / \mu_{g}}\right) \quad \alpha_{3}=-u_{0} .
\end{aligned}
$$

The dummy cell values are adapted to incorporate the presence of multiple droplets compared to a single fluid particle in the $\mathrm{H}-\mathrm{R}$ solution where in a first step the solution at $\theta=0$ has been prescribed along the entire lateral boundary. As FS3D employs a staggered grid arrangement 
the analytical solution is transformed into Cartesian coordinates with a corresponding transformation matrix and the definition $r=\sqrt{x_{i}^{2}+y_{j}^{2}+z_{k}^{2}}$ and $\theta=\cos ^{-1}\left(z_{k}^{2} / r\right)$ with $x_{i}, y_{j}, z_{k}$ being the corresponding distances to construct the values in the associated $u, v, w$-control volume of the two additional layers of cells at the lateral boundaries.

For the analysis of the influence of different lateral domain distances $y / D_{0}=z / D_{0}$ on the droplet stream the velocity profiles for the currently implemented boundary conditions in FS3D have been analyzed in a first step. Figure 2 shows the nondimensional velocity profiles $u / u_{0}$ at the axial location $x / D_{0}=8.5$ in the direction of motion. Results at other axial locations or along the $z$-axis yielded similar results and are not shown. The examined domain widths range from

(a)

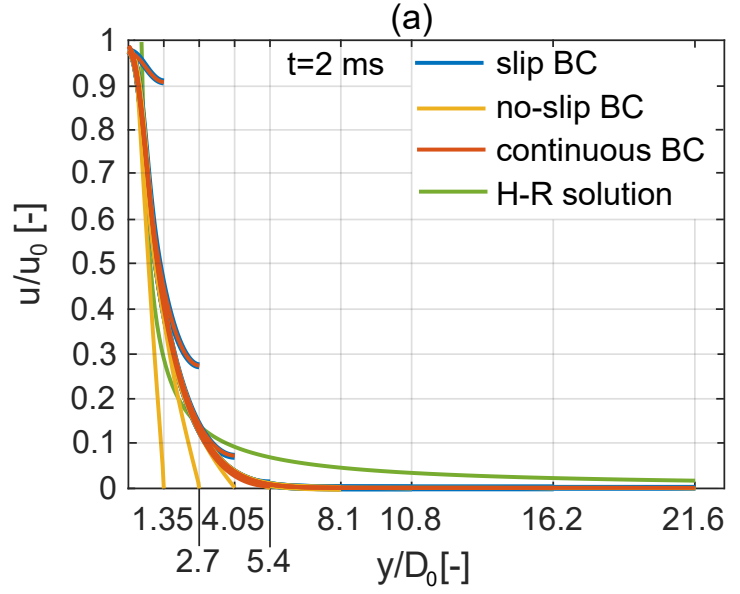

(b)

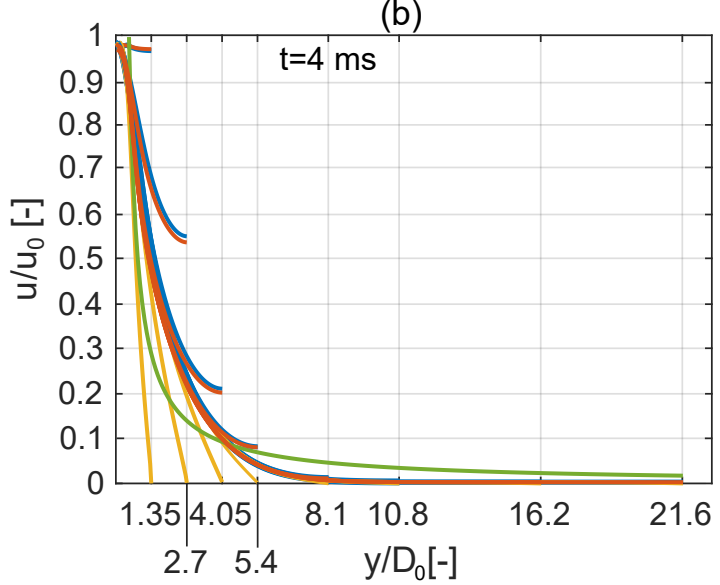

Figure 2. Velocity profiles of different boundary conditions for different lateral domain widths $y / D_{0}=z / D_{0}$ at $t=2 \mathrm{~ms}$ (a) and $t=4 \mathrm{~ms}$ (b) with the analytical H-R solution (green).

$y / D_{0}=1.35$ to 21.6 . It is worth noting that due to further improvement in the numerical setup the drift of the droplet stream towards the side boundaries could be minimized and therefore only half of the velocity profiles are plotted for the sake of clarity. Two distinguished times are selected to investigate the development of $u / u_{0}$ over the simulation duration. Figure 2 (a) at $t=2 \mathrm{~ms}$ represents the initiation of droplet grouping whereas (b) at $t=4 \mathrm{~ms}$ is closer to the coalescence of the droplets. The plot colors indicate the applied boundary conditions with the $\mathrm{H}-\mathrm{R}$ solution as reference. Two aspects, which are important to emphasize, are on the one hand the deviation of all velocity profiles from the analytical solution for all domain widths. For
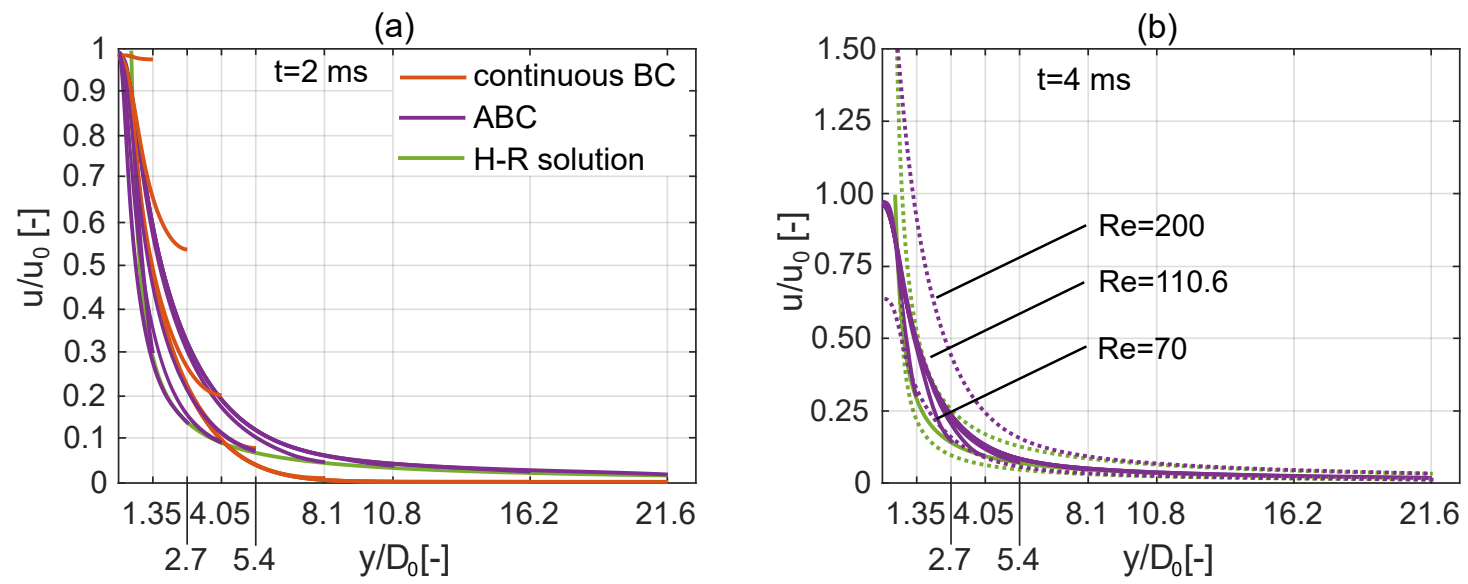

Figure 3. Velocity profiles of proposed ABCs for different lateral domain widths $y / D_{0}=z / D_{0}$ at $t=2 \mathrm{~ms}(\mathrm{a})$ and $t=4 \mathrm{~ms}$ (b). Dashed lines represent the solution for different Reynolds numbers.

$y / D_{0}<4.05$ velocities at the boundary edges lie above the free stream velocity (taken from the analytical $\mathrm{H}-\mathrm{R}$ solution) whereas for larger domains the velocity profiles tend to zero. On the 
other hand a rise of the free stream velocity of the prescribed boundaries over time is visible when considering figure 2 (b) at $t=4 \mathrm{~ms}$ especially for $y / D_{0}<8.1$ which indicates an accumulation of velocities in the computational domain. Even when increasing the lateral distance for the implemented BCs the free stream behavior of the velocity profiles is not accurately reproduced, additionally increasing computational costs. Moreover, the applied no-slip boundary conditions do not accurately describe grouping behavior in an unconfined environment in general, despite stabilizing the droplet stream.

The velocity profiles of the artificial boundary conditions depicted in figure 3 (a) (purple lines) reproduce the velocity decay and accurately represent the far field behavior especially for small domains widths $y / D_{0} \leq 5.4$. Even for the entire duration of the simulation no accumulation nor increase of the velocities near the lateral boundaries can be observed while the velocities at the domain edge approach the prescribed analytical free stream value smoothly (figure 3 (a) and (b)). Furthermore, due to the dissipated energy in terms of shear and pressure drag acting on the droplet stream the droplet velocity of the stream decreases which is indicated by a dimensionless velocity at $y / D_{0}=0$ of less than one, in accordance to the experimental results. For Reynolds numbers larger or less than $R e=110.6$ the free stream velocity at the prescribed lateral boundaries increases or decreases, respectively, in agreement to the analytical $\mathrm{H}-\mathrm{R}$ solution (figure 3 (b) dashed lines). The same trend of the velocity profiles approaching the free stream velocity as well as the far field decay behavior can be observed for simulations at different $R e$ numbers indicating a correct implementation of the artificial boundary conditions.

\section{Grouping Behavior}

To quantify the physical influence of the lateral boundaries on the droplet stream the grouping behavior is compared to available experimental data by Roth et al. [4]. Figure 4 (a) shows the change of inter droplet distance nondimensionalized by the group distance $a_{i} / g$ over time $t$ until final coalescence of the droplets (indicated by the dashed blue line; droplet distance falls below the droplet diameter $D_{0}$ ). The lateral domain boundaries for the depicted simulation are

(a)

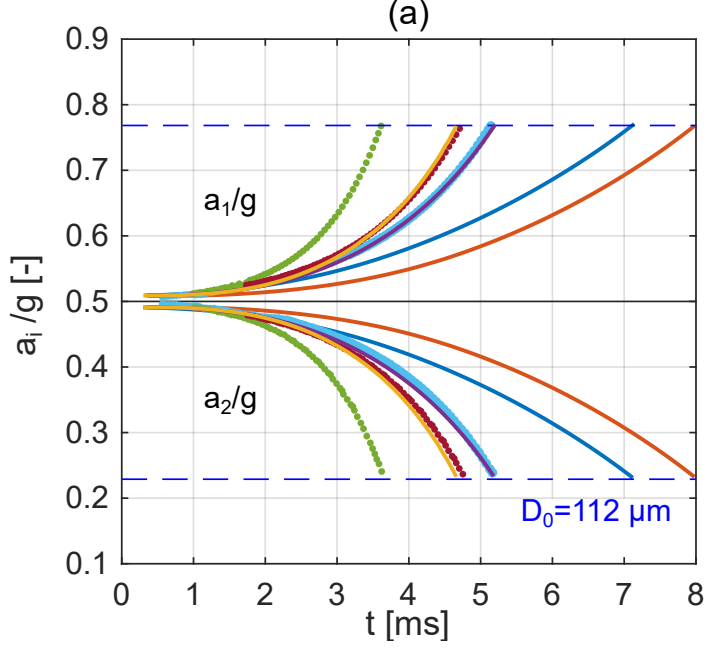

(b)

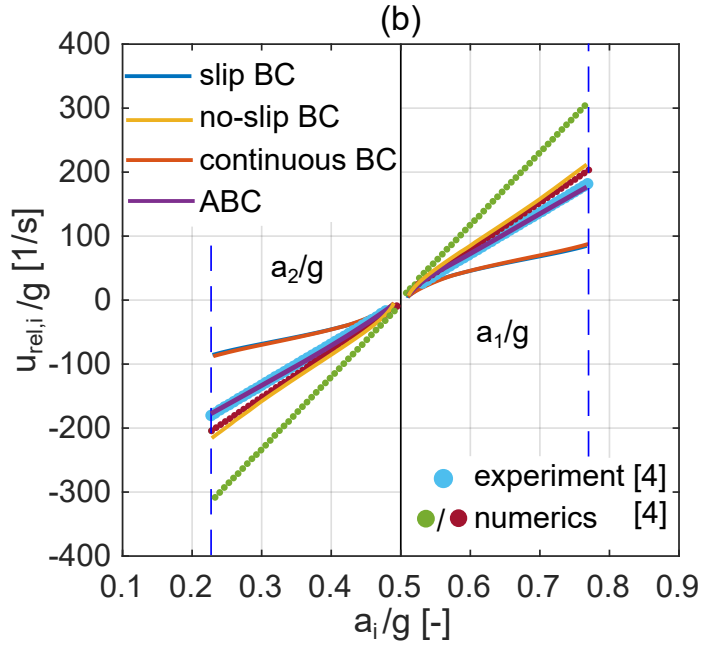

Figure 4. Evolution of inter droplet spacing $a_{i} / g$ as a function of time $t$ (a) and relative velocities $u_{r e l, i} / g$ as a function of relative spacing $a_{i} / g(\mathrm{~b})$ for different boundary conditions at $y / D_{0}=z / D_{0}=5.4$.

set to $y / D_{0}=z / D_{0}=5.4$. For the simulation with no-slip boundaries the grouping time is overestimated. This is expected as the confinement of the droplet stream leads to faster grouping due to different pressure distribution compared to a droplet in a free surrounding. For the slip and continuous BCs grouping arises slower than the experiments indicating that the prescribed boundaries alter the physical behavior of droplets in the stream at these lateral domain distances. For the implemented ABCs, $a_{i} / g$ fits accurately the experimental data. The trend of over- or underestimating droplet grouping can be directly assessed by plotting the relative velocities $u_{i, r e l} / g$ over the relative distance $a_{i} / g$ as seen in figure 4 (b). A linear relationship is 
obtained by substituting the time by the relative distances which can be regressed with an exponential function. A steeper slope (represented by the dotted green and red curve of numerical results by Roth and colleagues and the no-slip BC) indicates faster droplet grouping whereas a curve below the experimental data suggests a longer time required until grouping occurs and droplets coalesce. For domain sizes less than $y / D_{0}=5.4$ grouping behavior deviates from the experimental values for all prescribed boundary conditions, also for the newly implemented ABCs. The curves collapse for all boundary conditions with domain widths $y / D_{0} \geq 10.8$.

Additional simulations were performed to test the implementation of the proposed ABCs for different Reynolds numbers. Figures 5 (a) and (b) depict the grouping behavior compared to the original experiment for the implemented artificial boundaries. For the first case, a Reynolds

(a)

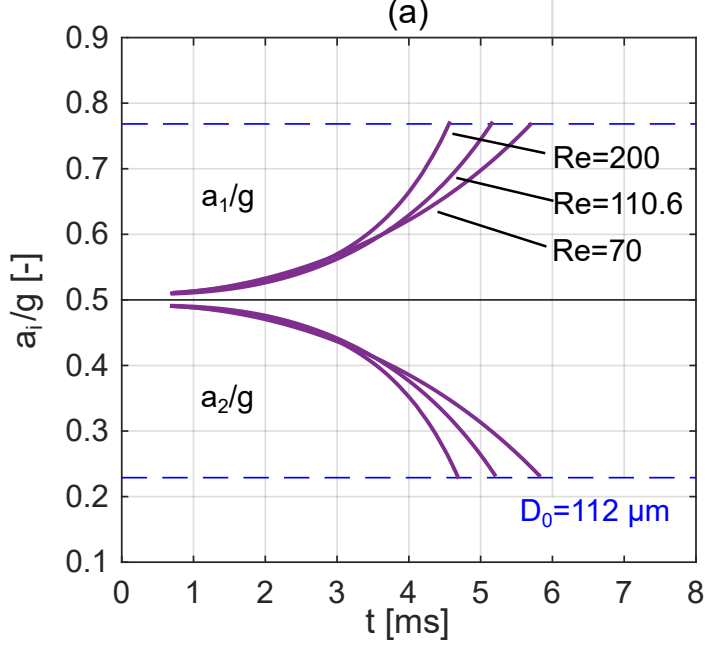

(b)

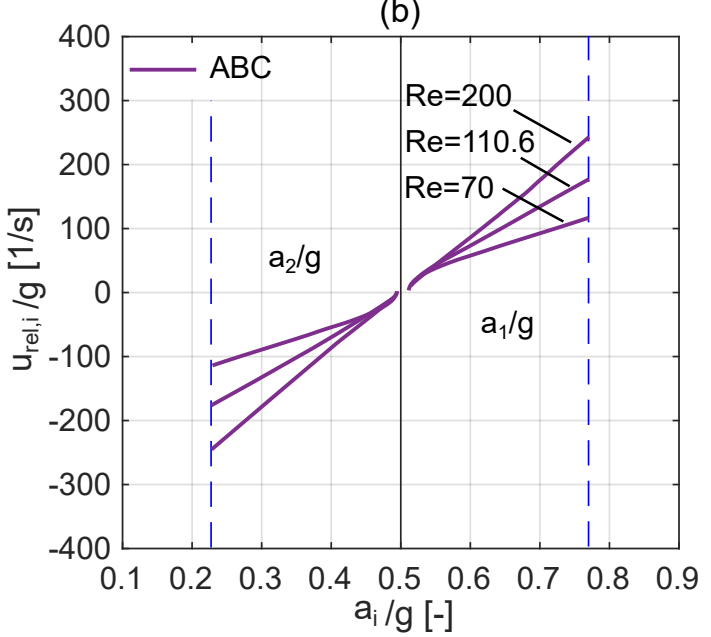

Figure 5. Grouping effects using $\mathrm{ABCs}$ for $R e=70, R e=110.6$ and $R e=200$. Evolution of inter droplet spacing $a_{i} / g$ as a function of time $t$ (a) and relative velocities $u_{r e l, i} / g$ as a function of relative spacing $a_{i} / g$ (b).

number of $R e=70$ is realized which results in an increase of time until droplet coalescence. Small disturbance within the system introduced by the different initial inter droplet distances in the droplet stream are amplified more slowly. The higher Reynolds number case $R e=200$ expedites the collision of droplets within the droplet stream. This stems from the fact of a developing asymmetric wake at these Reynolds numbers inducing a more irregular flow field. However, the exponential behavior for the change of inter droplet distance $a_{i} / g$ or relative velocities $u_{i, r e l} / g$ is observed for all simulations which is also in accordance to experimental results.

\section{Conclusions}

In this paper the influence of different boundary conditions on the grouping behavior in droplet streams was investigated with the multiphase DNS code FS3D. Therefore, different distances to the lateral domain were simulated and results were compared to available experimental data additionally analyzing velocity profiles. The implemented boundary conditions in combination with periodicity in the direction of droplet motion led to an accumulation of velocity with domains $y / D_{0}<10.8$ and did not represent the far field behavior in free streams correctly. For larger domains the grouping effect could be reproduced according to the experimental results, however, increasing computational costs. Artificial boundary conditions based on the analytical $\mathrm{H}-\mathrm{R}$ solution were implemented and expanded for the use of droplet streams while reproducing the decay of velocities in the far field accurately. The domain width can be reduced compared to the implemented BCs without physically influencing the grouping behavior in the droplet stream. Additionally, simulations with different Reynolds numbers were performed to show the suitability of the $A B C s$ revealing different times until droplet coalescence. With this reliable numerical framework further investigations concerning factors that influence grouping behavior can be performed. 


\section{Acknowledgements}

This work was funded by Deutsche Forschungsgemeinschaft (DFG, German Research Foundation) under Germany's Excellence Strategy - EXC 2075 - 390740016. We also acknowledge the support by the Stuttgart Center for Simulation Science (SimTech). Furthermore, the authors kindly acknowledge the High Performance Computing Center Stuttgart (HLRS) for the support and the supply of computational resources on the HPE Apollo (Hawk) platform under the Grant No. FS3D/11142 and the financial support of DFG through the project WE2549/41-1.

\section{Nomenclature}

Latin Symbols

a inter droplet distance [m]

$D \quad$ droplet diameter [m]

$\mathbf{f}_{\gamma} \quad$ surface tension force $\left[\mathrm{N} \mathrm{m}^{-3}\right]$

$f \quad$ VOF variable [-]

$\mathrm{g} \quad$ gravitational acceleration $\left[\mathrm{m} \mathrm{s}^{-2}\right]$

$g \quad$ distance between droplet groups [m]

$p \quad$ pressure $\left[\mathrm{N} \mathrm{m}^{-2}\right]$

$R \quad$ droplet radius [m]

$r \quad$ radial distance [m]

$t \quad$ time [s]

$\mathbf{u} \quad$ velocity vector $\left[\mathrm{m} \mathrm{s}^{-1}\right.$ ]

$u, v, w$ velocity components $\left[\mathrm{m} \mathrm{s}^{-1}\right]$

$x, y, z$ spatial coordinates [m]

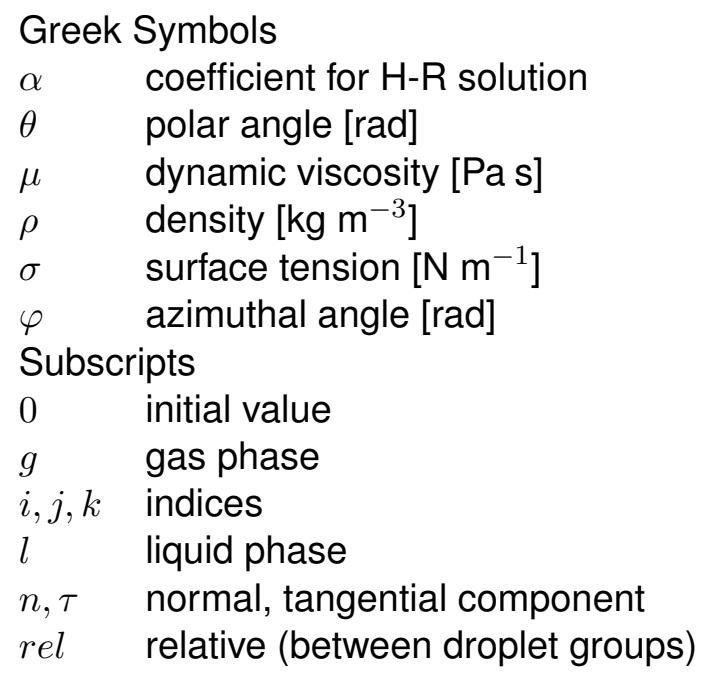

\section{References}

[1] Sirignano, W. A., 2014, "Fluid dynamics and transport of droplets and sprays", Cambridge University Press.

[2] Katoshevski, D., 2006, Aerosol Air Quality Research, 6(10), pp. 54-66.

[3] Roth, N., Weigand, B., Katoshevski, D., Greenberg, J.B., May 29. 2015, DIPSI Workshop 2015 on Droplet Impact Phenomena \& Spray Investigations.

[4] Roth, N., Gomaa, H., Livne, A., Katoshevski, D., Weigand, B., Sep. 6.-8. 2017, 28th European Conference on Liquid Atomization and Spray Systems.

[5] Alke, A., Bothe, D., Kröger, M., Weigand, B., Weirich, D., Weking, H., March 2010, ERCOFTAC Bulletin, 82, pp. 5-10.

[6] Hirt, C.W., Nichols, B.D., 1981, J. Comput. Phys., 39 (1), pp. 201-225.

[7] Rider, W. J., Kothe, D. B., 1998, J. Comput. Phys., 141 (2), pp. 112-152.

[8] Lafaurie, B., Nardone, C., Scardovelli, R., Zaleski, S., Zanetti, G., 1994, J. Comput. Phys., 113 (1), pp. 134-147.

[9] Ren, W., Reutzsch, J., Weigand, B., 2020, Fluids, 5 (3), 158.

[10] Fest-Santini, S., Steigerwald, J., Santini, M., Cossali, G.E., Weigand, B., 2021, Computers \& Fluids, 214, 104761.

[11] Baggio, M., Weigand, B., Sep. 2.-4. 2019, 29th European Conference on Liquid Atomization and Spray Systems.

[12] Reutzsch, J., Kieffer-Roth, C., Weigand, B., 2020, J. Comput. Phys., 413, 109455.

[13] Kaufmann, J., Geppert, A., Ertl, M., Bernard, R., Vaikuntanathan, V., Lamanna, G., Weigand, B., July 22.-26. 2018, 14th Triennial International Conference on Liquid Atomization and Spray Systems.

[14] Eisenschmidt, K., Ertl, M., Gomaa, H., Kieffer-Roth, C., Meister, C., Rauschenberger, P., Reitzle, M., Schlottke, K., Weigand, B., 2016, Appl. Math. a. Comp., 272 (2), pp. 508-517.

[15] Weirich, D., Köhne, M., Bothe, D., 2014, Int. J. Numer. Meth. Fluids, 75 (3), pp. 184-204. 